\title{
Evolución de la biosíntesis de lípidos durante la maduración de las variedades de aceituna"Picual" y "Gordal"
}

\author{
Por J.M. García y M. Mancha \\ Instituto de la Grasa y sus Derivados. (CSIC). \\ Avda. Padre García Tejero, 4 - 41012 Sevilla. España.
}

\section{RESUMEN}

Evolución de la biosíntesis de lípidos durante la maduración de las variedades de aceituna "Picual" y "Gordal"

Se ha estudiado comparativamente la evolución de la biosíntesis de lípidos durante la maduración de dos variedades de aceituna (Olea europaea cv. "Picual" y "Gordal"), mediante la medida de la incorporación de ${ }^{14} \mathrm{C}$-acetato a los lípidos del tejido intacto del fruto. La variedad "Picual" presentó mayor actividad de síntesis lipídica que la variedad "Gordal". En la primera, el máximo de actividad tuvo lugar durante los meses de septiembre y octubre, mientras que en la segunda ocurrió entre agosto y septiembre. En ambas variedades la incorporación en lipidos polares fue previa a la incorporación a triglicéridos y algo más intensa. La incorporación a diglicéridos y ácidos grasos libres fue menor y con grandes oscilaciones.

PALABRAS-CLAVE: Aceituna - Lípido (biosíntesis) - Maduración

\section{SUMMARY}

Evolution of the lipids biosynthesis during the maturation of the olive varieties "Picual" and "Gordal".

The evolution of lipid biosynthesis during the ripening period of two varieties of olive fruits (Olea europaea cv. "Picual" and "Gordal") has been compared by measuring the incorporation of ${ }^{14} \mathrm{C}$-acetate into lipids of intact exocarp tissue. The variety "Picual" showed a higher activity of lipid synthesis than the variety "Gordal". In the former the maximal activity took place during september and october. In the latter it ocurred during august and september. In both varieties the incorporation into polar lipids preceeded the incorporation into triacylglycerols the former being slightly more intense. The incorporation into diacylglycerols and free fatty acids was low and variable.

KEY-WORDS: Lipid (biosynthesis) - Maturation - Olive.

\section{INTRODUCCION}

La incorporación de ${ }^{14} \mathrm{C}$-acetato en los lípidos de un tejido vegetal, puede constituir una buena estimación de su capacidad de biosíntesis lipídica, pues refleja la actuación de la maquinaria enzimática responsable del proceso anabólico de los lípidos, desde la carboxilación del acetato (Nikolau y Hawke, 1984), y la formación de los diferentes ácidos grasos dentro del plastidio (Stumpf, 1988), hasta la formación de los glicerolípidos por acción de las diversas aciltransferasas (Slack y Browse, 1984 y Stobart y Stymne, 1985).

Si se estudia como evoluciona la incorporación de este precursor en los lípidos de un determinado tejido a lo largo de la maduración, se obtiene una curva caracte- rística y propia para dicho tejido en función de las condiciones de cultivo en que se desarrolló. Estudios de este tipo, permiten comparar el previsible comportamiento de diferentes especies o variedades bajo similares condiciones de cultivo. A este respecto, se han realizado trabajos en semillas de soja (García et al., 1988) y de Girasol (Mancha et al., 1985). En aceituna, Mancha (1978) describió la síntesis de lípidos de la pulpa y Marzouk y Cherif (1980) realizaron estudios sobre la evolución de la capacidad de biosíntesis lipídica en la variedad norteafricana "Chemlali". Posteriormente, Daza y Donaire (1982) estudiaron la evolución de la incorporación de ${ }^{14} \mathrm{C}$-acetato en los lípidos de oleosomas obtenidos durante la maduración de la variedad "Marteña". Más recientemente Sánchez et al., (1990) pusieron de manifiesto el efecto de la luz y de la temperatura sobre la incorporación de ${ }^{14} \mathrm{C}$ acetato en los lípidos de tejido intacto de aceituna.

En el presente trabajo se realiza un estudio comparado de la biosíntesis lipídica durante la maduración de dos variedades diferentes de aceituna, la variedad "Picual" de gran importancia para la industria almazarera, dado el elevado rendimiento que ofrece en la obtención de aceite y la variedad "Gordal", básicamente utilizada para el consumo de mesa. El objetivo primario es observar si las diferencias en el contenido final de aceite (25\% para "Picual" y $19 \%$ para "Gordal"), se deben a un diferente modelo de incorporación de ${ }^{14} \mathrm{C}$-acetato en los distintos lípidos durante el proceso de maduración. Así mismo, el conocimiento de la evolución de la capacidad de biosíntesis lipídica permite identificar el momento en que ésta se interrumpe en el fruto. Dato que conviene tener presente a la hora de decidir el momento idóneo de cosecha, ya que a partir de entonces no se producirá incremento alguno en el contenido lipídico de la aceituna (Marzouk y Cherif, 1981). Como cualquier otro fruto, la aceituna va perdiendo viabilidad de conservación conforme avanza su maduración, al debilitarse la dureza de sus paredes celulares y al hacerse progresivamente más sensible a los daños mecánicos y a las infecciones por microorganismos parásitos (Streif, 1983). Por consiguiente, la cosecha debe realizarse lo más temprano posible, una vez que se ha llegado al máximo de acumulación lipídica y cuando las características sensoriales del aceite obtenido sean aceptables para el consumo. 


\section{PARTE EXPERIMENTAL}

\subsection{Material biológico}

Se han utilizado dos variedades de aceituna (Olea europaea cv. "Picual" y "Gordal") suministradas por el EUITA de Sevilla, habiéndose realizado una toma de muestras y un ensayo semanal entre junio y diciembre. Se pesan 25 aceitunas por ensayo y variedad, se establece su peso medio y se seleccionan 5 aceitunas, realizándose con cada una de ellas una incubación con ${ }^{14} \mathrm{C}$ acetato.

\subsection{Incorporación de ${ }^{14} \mathrm{C}$-acetato}

Las aceitunas deshuesadas se incubaron a $25^{\circ} \mathrm{C}$ durante 4 horas en oscuridad y con agitación, con ${ }^{14} \mathrm{C}$-acetato (disolución acuosa de $0,5 \mu \mathrm{Ci} / \mathrm{ml}$ ), con un volumen total en $\mathrm{ml}$ igual al doble del peso medio del fruto expresado en gramos. La reacción fue interrumpida hirviendo la muestra durante 10 minutos, eliminándose el precursor radiactivo mediante lavados con agua destilada. Finalmente la muestra, limpia y seca, se conservó a $-40^{\circ} \mathrm{C}$ hasta su procesamiento.

\subsection{Extracción de lípidos}

Se ha utilizado el método de Hara y Radin (1978) que usa la mezcla de hexano:isopropanol $(3: 2, v / v)$. El extracto de lípidos totales se disolvió en $1 \mathrm{ml}$ de mezcla hexano:isopropanol $(7: 2, \mathrm{v} / \mathrm{v})$ y se conservó a $-40^{\circ} \mathrm{C}$ en tubo cerrado.

\subsection{Fraccionamiento de lípidos y cuantificación} de la radiactividad incorporada

Los lípidos extraídos se separaron por cromatografía en capa fina (CCF) en placas de gel de sílice de 0,25 $\mathrm{mm}$ desarrolladas con una mezcla de hexano: éter etílico: ácido fórmico $(75: 25: 1, \mathrm{v} / \mathrm{v} / \mathrm{v})$, usando como mezcla patrón el resultado de una hidrólisis con lipasa pancreática de un aceite refinado de oliva. Se obtuvieron cinco bandas que corresponden a otras tantas clases lipídicas: hidrocarburos y ceras; triglicéridos; ácidos grasos libres; diglicéridos y en el origen, los lípidos polares (fosfolípidos, galactolípidos y sulfolípidos).

La incorporación de ${ }^{14} \mathrm{C}$ a los lípidos totales se cuantificó por duplicado tomando una alicuota y midiendo la radiactividad en contador de centelleo líquido rackbeta (LKB). La distribución de la radiactividad entre las diferentes clases de lípidos se determinó, tras su separación en CCF, mediante un analizador lineal de radiactividad Berthold.

\subsection{Tratamiento estadístico de los resultados}

A todos los datos se les realizó el análisis de la varianza. La diferencia entre las medias se analizó mediante el test de Duncan, por el que se determinó, en cada caso, la diferencia significativa mínima (LSD) con un nivel de significación del $5 \%$.

\section{RESULTADOS Y DISCUSION}

Durante todo el proceso de maduración del fruto la incorporación de ${ }^{14} \mathrm{C}$-acetato en la fracción de lípidos totales del tejido intacto fue significativamente mayor en la aceituna "Picual" que en la aceituna "Gordal" (fig. 1). En la variedad "Picual", se inició un aumento exponencial de la síntesis de lípidos en la sexta semana (principios de agosto), llegando a ser máxima en la decimocuarta semana (mediados de septiembre), para descender rápidamente cuatro semanas más tarde. En la variedad "Gordal", no se observó un incremento tan nítido, alcanzándose los valores máximos dos semanas antes que en la aceituna "Picual". Posteriormente, hubo una caída de actividad, asi mismo gradual, hasta prácticamente desaparecer a comienzos de diciembre (vigesimotercera semana).

Daza y Donaire (1982) observaron que el máximo de incorporación de ${ }^{14} \mathrm{C}$-acetato en los oleosomas de aceitunas de la variedad "Marteño" tenía lugar en septiembre, coincidiendo con lo observado en la variedad "Picual". Marzouk y Cherif (1980) encontraron que el máximo de incorporación en la variedad "Chemlali" se producía en la decimoprimera semana de maduración del fruto, aproximadamente como ocurriera con la variedad "Gordal".

Las variedades especializadas en la producción de aceite como "Picual", "Marteño" o "Chemlali" presentan igualmente una incorporación en lípidos totales que se

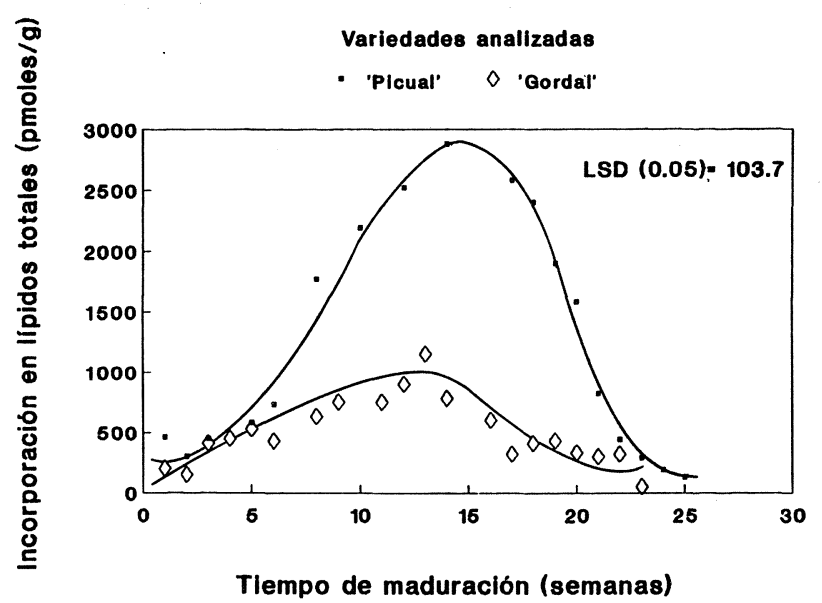

Figura 1

Evolución de la incorporación de ${ }^{14} \mathrm{C}$-acetato en la totalidad de los lípidos durante la maduración de las variedades de aceituna "Picual" y "Gordal".

mantiene con valores relativamente elevados entre la décima y la vigésima semanas.

El perfil de la evolución de la incorporación del precursor radiactivo en los lípidos polares fue muy diferente en ambas variedades (fig. 2). La variedad "Picual", presentó un máximo coincidiendo con el observado para los lípidos totales. En cambio, el máximo de incorporación a lípidos polares fue anterior en la variedad "Gordal", apareciendo antes de la décima semana (mediados de agosto). En la decimocuarta semana, coincidiendo aproximadamente con el máximo de incorporación en la aceituna "Picual", se inició un significativo descenso de la incorporación, que terminó desapareciendo en diciembre. En cualquier caso, salvo en la fase inicial del ensayo, en que los valores de incorporación fueron similares, la variedad "Picual" desarrolló una actividad biosintética significativamente superior. Otros autores (Marzouk y Cherif, 1980; Daza 
y Donaire, 1982) han observado en otras variedades de aceitunas, que el máximo de incorporación de ${ }^{14} \mathrm{C}$-acetato en lípidos polares se desarrollaba durante las primeras semanas del desarrollo del fruto, como ocurre en la variedad "Gordal". Marzouk y Cherif (1980) detectaron en la variedad "Chemlali" una rápida caída posterior en la incorporación en este tipo de lípidos hasta casi anularse a partir de la décima semana de maduración.

La incorporación de ${ }^{14} \mathrm{C}$-acetato en diglicéridos fue muy superior en la variedad "Picual" (fig. 3). Sin embargo, ambas presentan sucesivos altibajos, debido, probablemente, al carácter de intermediarios metabólicos de estos lípidos, al ser los diglicéridos paso obligado para la síntesis de triglicéridos (Gurr, 1980) o galactolípidos (Douce y Joyard, 1980). Su síntesis en un momento dado en el tejido intacto depende tanto de las actividades que determinan su formación como de las que lo utilizan como sustrato. Ello podría explicar las irregularidades en las curvas que describen su biosíntesis a lo largo de la maduración del fruto.

La incorporación en ácidos grasos libres fue también muy diferente en las dos variedades estudiadas. Hasta la décima semana los valores de incorporación fueron bajos (36,4 $\pm 9,9$ pmoles/g) en ambas variedades. Sin embargo, mientras que en la variedad "Gordal" éstos valores se mantuvieron hasta el final de la maduración, en la "Picual" se observó, a partir de la décima semana, un incremento de la incorporación con grandes altibajos $(116,9 \pm 52,7$ pmoles $/ g)$. Probablemente, la presencia de ácidos grasos libres se debe a la actividad aciltioesterasa sobre los acil-CoAs (Mancha y García, 1982). Menos plausible sería que el aumento en el porcentaje de incorporación en ácidos grasos se debiera a un incremento de la actividad lipolítica. Normalmente, salvo que el fruto esté muy maduro, la aparición de la actividad lipásica tiene

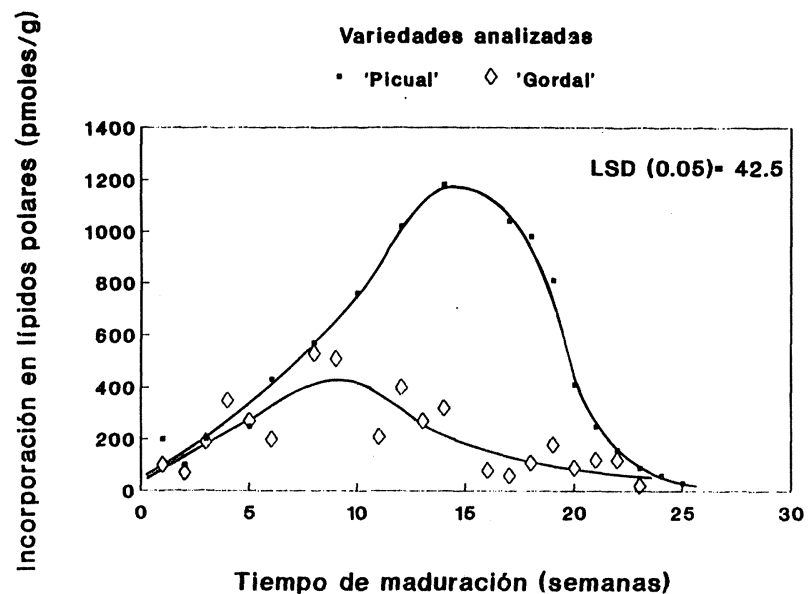

Figura 2

Evolución de la incorporación de ${ }^{14} \mathrm{C}$-acetato en los lípidos polares durante la maduración de las variedades de aceituna "Picual" y "Gordal"

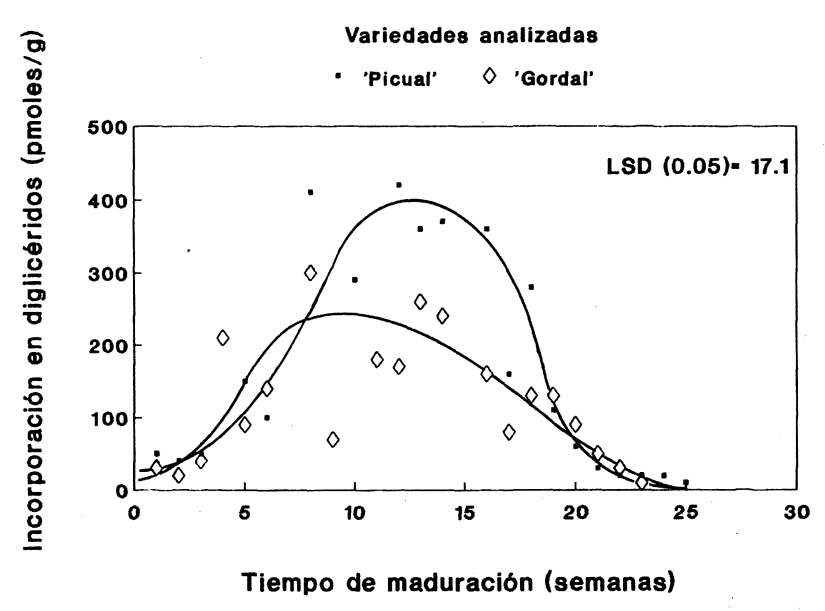

Figura 3

Evolución de la incorporación de ${ }^{14} \mathrm{C}$-acetato en los diglicéridos durante la maduración de las variedades de aceituna "Picual" y "Gordal"

lugar después de la recolección de la aceituna, como lo demuestra el hecho de que los aceites procedentes de aceitunas recién cosechadas o conservadas a $5^{\circ} \mathrm{C}$, incluso durante un mes, presenten un grado de acidez mínimo (datos no publicados). Si el aumento de la incorporación en ácidos grasos libres se debiera a una actividad lipásica, dada la enorme cantidad de glicerolípidos presentes en la aceituna madura, se debería observar, al mismo tiempo, un aumento notable del grado de acidez en el aceite extraído de estas aceitunas, dado que la proporción el glicerolípidos marcados con ${ }^{14} \mathrm{C}$ es muy pequeña con respecto al total. En cambio la más elevada proporción de acil-CoAs marcados frente a los no marcados, sí podría justificar la recuperación de una notable cantidad de ácidos grasos libres marcados debido a la actividad acil-CoA tioesterasa sin que se registrara un aumento sensible de la acidez del aceite.

En la figura 4 se representa la evolución de la incorporación en los triglicéridos. Puede observarse como el perfil de las curvas es similar a las obtenidas con los lípidos polares, si bien en esta ocasión los máximos de actividad de ambas variedades están más próximos con una diferencia de sólo dos semanas. Este acercamiento se debió a que el máximo en la variedad "Gordal" apareció 4 semanas más tarde en los triglicéridos que en los lípidos polares, mientras que en la variedad "Picual" ambos máximos estuvieron muy próximos.

En las variedades "Chemlali" y "Marteño" los máximos de sintesis de triglicéridos y lípidos polares se hallan más separados incluso que en la variedad "Gordal" (Marzouk y Cherif, 1980; Daza y Donaire, 1982). Este hecho se ha interpretado asociando el temprano ascenso en la incorporación en lípidos polares con la formación de membranas que posteriormente se emplearán para contener los triglicéridos que se sintetizarán a continuación. En la 
variedad "Picual" hay un claro solapamiento de ambos procesos. La formación de membranas y la acumulación de lípidos de reserva se realizaría más simultánea que consecutivamente.

Por otra parte, los estudios de cinética de incorporación y de pulso y captura en tejido intacto de semilla de soja en formación, incubado con ${ }^{14} \mathrm{C}$-acetato, revelan que éste, en principio fijado principalmente en los lípidos polares, sobre todo en fosfatidilcolina, termina incorporándose en los triglicéridos pertenecientes a la fracción oleosómica, que contiene a los orgánulos encargados del almacenamiento de los lípidos de reserva en las plantas (García et al., 1988). Por tanto, no sería correcto desligar por completo la incorporación recuperada en lípidos polares con la formación de lípidos de reserva.

La biosíntesis lipídica se interrumpe prácticamente en la vigesimoquinta semana en la variedad "Picual" y dos semanas antes en la variedad "Gordal". Desde el punto de vista de la producción de aceite éste sería el momento más adecuado para el inicio de la cosecha. Sólo una mejora de las características organolépticas del aceite o la falta de disponibilidad de la industria extractora podrían justificar el mantenimiento del fruto en el árbol, en donde progresivamente irá perdiendo su resistencia a los daños mecánicos y a la parasitación.

\section{CONCLUSIONES}

La variedad "Picual" demostró ser más activa que la "Gordal" en la biosíntesis de todas las clases de lípidos. La incorporación del precursor respondió a la diferente capacidad de síntesis de ambas variedades.

Fue diferente el período en que se registró la actividad máxima de biosíntesis de lípidos en ambas variedades. Posiblemente como reflejo de un diferente acopla-
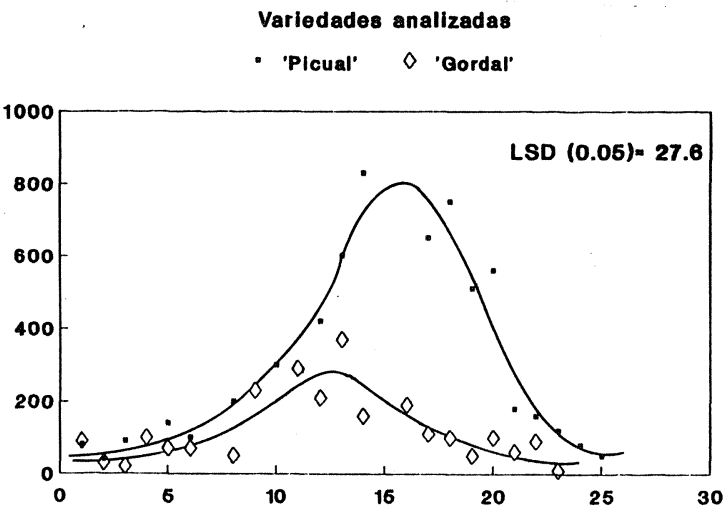

Tiempo de maduración (semanas)

Figura 4

Evolución de la incorporación de ${ }^{14} \mathrm{C}$-acetato en los triglicéridos durante la maduración de las variedades de aceituna "Picual" y "Gordal" miento entre la formación de estructuras membranosas y la acumulación de lípidos de reserva.

La biosíntesis lipídica se interrumpe a las 23 semanas de maduración en la variedad "Gordal" y a las 25 semanas en la variedad "Picual", siendo este momento, desde el punto de vista exclusivamente de la producción de aceite, aconsejable para el inicio de la cosecha.

\section{AGRADECIMIENTOS}

Agradecemos muy encarecidamente a D. ํ María del Carmen Ruiz Bayona su asistencia técnica.

\section{BIBLIOGRAFIA}

Daza, L.M. y Donaire, J.P. (1982).- "Composition and lipid biosynthesis 'in vivo' in oil bodies of olive tree fruits". En "Biochemistry and metabolism of plant lipids".- Wintermans y Kuiper, eds. pp. 237-241. Elsevier Biomedical Press, Amsterdam.

Douce, R. y Joyard, J. (1980).- "Plant galactolipids". En "Lipids: Structure and Function" Vol. 4 de la colección: "The Biochemistry of Plants. A comprehensive treatise".- pp. 321-362. Academic Press. NY. USA.

Garcia, J.M.; Quintero, L.C. y Mancha, M. (1988).- "Oil bodies and lipid synthesis in developing soybean seeds".- Phytochemistry. 27, 3.0833.087.

Gurr, M.I. (1980).- "The biosynthesis of triacylglycerols. En "Lipids: Structure and Function" Volumen $4 .^{\circ}$ de la colección: "The Biochemistry of Plants. A comprehensive treatise".- pp. 205-249. Academic Press. NY. USA.

Hara, A. y Radin, N.S. (1978).- "Lipid extraction of tissues with a low toxicity solvent".- Anal. Biochem. 90, 420-426.

Mancha, M. (1978).- "Lípidos sintetizados por la pulpa de aceituna a partir de precursores marcados con carbono-14".- Grasas y Aceites 29, 263-268.

Mancha, M. y Garcia, J.M. (1982).- "Acyl-Coa Synthetase, Acyltransferase and Acyl-Coa Thioesterase activities of oil bodies from Avocado Mesocarp". En "Biochemistry and metabolism of plant lipids".- Wintermans, J.F.G.M. y Kuiper, P.J.C. eds., pp. 243-246.- Elsevier Biomedical Press. Amsterdam.

Mancha, M.; Garcia, J.M. y Quintero, L.C. (1985).- "Lipid biosynthesis and breeding in oil seeds". En "Proceedings of the Sixth Meeting of the Eucarpia (Section Oil and Protein Crops)".- Fernández Martínez, J. ed., pp. 83-91.- Dirección General de Investigación y Exłtensión Agraria. Junta de Andalucia.

Marzouk B. y Cherif, A. (1980).- "La lipogenèse dans l'olive III. Biosynthèse des lipides à partir de l'acetate-1-14-C.- Rev. Fr. Corps Gras. 27, 487-491.

Marzouk , B. y Cherif, A. (1981).- "La lipogenèse dans l'olive. I. Formation des lipides neutres".- Oléagineux 36, 77-82.

Nikolau, B.S. y Hawke, J.C. (1984).- "Purification and characterization of maize leaf acetyl-coenzyme A carboxilase".- Arch. Biochem. Biophys. 228, 86-96

Sánchez, J.; De la Osa, C. y Harwood, J.L. (1990).- "Effect of light and temperature on the biosynthesis of storage triacylglycerols in olive (Olea europeae) fruits". - En "Plant Lipid Biochemistry, Structure and Utilization".- Quin, P.J. y Harwood, J.L. eds., pp. 390-392.- Portland Press Ltd. London, UK.

Slack, C.R. y Browse, J.A. (1984).- "Synthesis of storage lipids in developing seeds". En "Seed Physiology".- Murray, D.R. ed., pp. 209-244.Academic Press, Sydney. Australia.

Stobart, A.K. y Stimne, S. (1985).- "The interconversion of diacylglycerol and phosphatidylcholine during triacylgliceron production in microsomal preparations of developing cotyledons of safflower (Carthamus tinctorius L.)".- Biochem. J. 232, 217-221.

Streif, J. (1983).- "Der optimale Erntetermin bein Apfel I. Qualitätsentwicklung und Reife".- Gartenbauwissenschaft 48, 154-159.

Stumpf, P.F. (1988).- "Plant lipid biotechnology through the looking glass". En "Proceedings of World Conference on Biotechnology for the Fats and Oils industry".- Applewhite, T.H., ed. pp. 1-6.- AOCS. Kraft Inc. Research \& Development. Illinois. USA.

(Recibido: Febrero 1992) 